\title{
Motion Control of Hyper Redundant Robots by Learning Control Based on Linear Combination of Error History and Initial Configuration Optimization with Backward Learning*
}

\author{
Daisuke MATSUURA** and Nobuyuki IWATSUKI** \\ ** Dept. of Mechanical Sciences and Engineering, Tokyo Institute of Technology \\ 2-12-1 Ookayama, Meguro-ku, Tokyo, 152-8552, Japan \\ E-mail:matsuurd@mep.titech.ac.jp
}

\begin{abstract}
This paper describes the motion control of hyper redundant robots using a learning control scheme based on linear combination of error history. The learning control scheme is formulated with three elements: general solution of inverse kinematics with pseudo inverse of Jacobian matrix to achieve main task, condition to achieve several subtasks and compensation by linear combination of obtained time history of output error. In order to make planar serial manipulator with hyper redundancy achieving main task, tracking of desired trajectory by its output link while performing obstacle avoidance as subtask, several subtask setting schemes to prevent from partially singular configuration caused by interference between main task and subtasks are proposed. The backward learning scheme is also proposed to obtain optimum initial configuration for the proposed learning control scheme. Several simulations and experiments with a planar 10R serial manipulator demonstrate the effectiveness of proposed control scheme.
\end{abstract}

Key words: Learning Control, Hyper Redundant Robot, Error History, Vortex Field, Obstacle Avoidance

\section{Introduction}

High performance robots that can be used in human daily environment should be intelligent and dexterous to generate complicated and flexible motions. Therefore, new robotic mechanisms having hyper redundant degrees of freedom (DOF) more than that of motion space will be strongly required. In order to determine joint input of such mechanisms, optimum control method is necessary, which takes account of not only utilizing of kinematical redundancy but also reducing output error caused by unexpected dynamical factors such as friction, non-linearity of actuator system and so on. As one of the effective solution for this problem, learning control method ${ }^{(1)-(4)}$ has been proposed. The authors also have applied linear learning control scheme ${ }^{(5),(6)}$ for the control problem of redundant robots ${ }^{(6),(7)}$. Especially in the reference paper [7], a planar serial manipulator obtained desired trajectories of its output link as a main task while avoiding two obstacles as subtasks. However, because those robots had just a few redundant DOF, the availability of the proposed control scheme on the application to hyper redundant robots has not been discussed. In general, when robots have hyper redundancy, they often generate unstable 
motion or take a partial singular configuration because of the interference of main task and subtask, then fail to accomplish given tasks ${ }^{(8)}$. This becomes a fatal problem to control hyper redundant robots when more subtasks are given to utilize their hyper redundant degrees of freedom effectively.

This paper thus discusses new effective methods to set subtasks to avoid the interference between main task and subtask for the proposed learning control scheme based on linear combination of error history. Then the improved control method with the new subtask setting scheme is validated with a $10 \mathrm{R}$ planar serial manipulator having hyper redundancy in a number of motion control simulations and experiments in complex environments.

2. Learning control based on linear combination of error history and its problems

\subsection{Learning control based on linear combination of error history}

Let's consider a redundant mechanism having $N$ DOF and $M$ outputs $(N>M)$. Output velocities of its output link $\dot{\boldsymbol{r}}_{\mathrm{m}}$ are set as main task and output velocities of the other links $\dot{\boldsymbol{r}}_{\mathrm{s}}$ are set as subtask. The joint input velocity $\dot{\boldsymbol{\theta}}$ that achieves the main task is written as

$$
\dot{\boldsymbol{\theta}}=\boldsymbol{J}_{\mathrm{m}}^{\#} \dot{\boldsymbol{r}}_{\mathrm{m}}+\left(\boldsymbol{I}-\boldsymbol{J}_{\mathrm{m}}^{\#} \boldsymbol{J}_{\mathrm{m}}\right) \dot{\boldsymbol{\theta}}_{0}
$$

where $\boldsymbol{J}_{\mathrm{m}}$ denotes Jacobian matrix of the main task and $\boldsymbol{J}_{\mathrm{m}}{ }^{\#}$ is its pseudo-inverse, given as $\boldsymbol{J}_{\mathrm{m}}{ }^{\#}=\boldsymbol{J}_{\mathrm{m}}{ }^{\mathrm{T}}\left(\boldsymbol{J}_{\mathrm{m}} \boldsymbol{J}_{\mathrm{m}}{ }^{\mathrm{T}}\right)^{-1}$. The second term on the right side of Eq.(1) adjusts the joint input velocity $\dot{\boldsymbol{\theta}}$ by an arbitrary vector $\dot{\boldsymbol{\theta}}_{0}$ without any obstruction of the main task achievement. Therefore $\dot{\boldsymbol{\theta}}_{0}$ can be used to achieve the subtask $\dot{\boldsymbol{r}}_{\mathrm{s}}$. As the first step of the obtaining of $\dot{\boldsymbol{\theta}}_{0}$, output velocity of subtask is written as

$$
\dot{\boldsymbol{r}}_{\mathrm{s}}=\boldsymbol{J}_{\mathrm{s}} \dot{\boldsymbol{\theta}}
$$

Where $\boldsymbol{J}_{\mathrm{s}}$ denotes Jacobian matrix of the subtasks. From Eqs.(1) and (2), the vector $\dot{\boldsymbol{\theta}}_{0}$ to achieve both $\dot{\boldsymbol{r}}_{\mathrm{m}}$ and $\dot{\boldsymbol{r}}_{\mathrm{s}}$ is determined as

$$
\dot{\boldsymbol{\theta}}_{0}=\left[\boldsymbol{J}_{\mathrm{s}}\left(\boldsymbol{I}-\boldsymbol{J}_{\mathrm{m}}^{\#} \boldsymbol{J}_{\mathrm{m}}\right)\right]^{\#}\left(\dot{\boldsymbol{r}}_{\mathrm{s}}-\boldsymbol{J}_{\mathrm{s}} \boldsymbol{J}_{\mathrm{m}}^{\#} \dot{\boldsymbol{r}}_{\mathrm{m}}\right)
$$

By substituting Eq.(3) into Eq.(1) and rewriting the result in difference formula, the joint input increment $\Delta \boldsymbol{\theta}$ which achieves both main and subtasks is given as

$$
\Delta \boldsymbol{\theta}=\boldsymbol{J}_{\mathrm{m}}^{\#} \Delta \boldsymbol{r}_{\mathrm{m}}+\left(\boldsymbol{I}-\boldsymbol{J}_{\mathrm{m}}^{\#} \boldsymbol{J}_{\mathrm{m}}\right)\left[\boldsymbol{J}_{\mathrm{s}}\left(\boldsymbol{I}-\boldsymbol{J}_{\mathrm{m}}^{\#} \boldsymbol{J}_{\mathrm{m}}\right)\right]^{\#}\left(\Delta \boldsymbol{r}_{\mathrm{s}}-\boldsymbol{J}_{\mathrm{s}} \boldsymbol{J}_{\mathrm{m}}^{\#} \Delta \boldsymbol{r}_{\mathrm{m}}\right)
$$

The desired trajectory of the main task is given as a series of desired position vector of output link, ${ }^{d} \boldsymbol{r}_{i}$, at the time $t=t_{i}(i=0,1,2, \ldots, I)$. Then iterative learning control scheme ${ }^{(5)}$ is applied to reduce output error of real robot caused by unexpected factors, e.g. frictions, non-linearity of motor output etc., and to utilize hyper redundancy enough effectively. An 'operation' is defined as a process to put the output link on its desired position at a time $t=t_{i}$ and a 'trial' is defined as a series of operations to achieve all of the desired motions. Three output error vectors at each combination of current and previous trials and operations are calculated as

$$
\boldsymbol{e}_{n-1, i}={ }^{d} \boldsymbol{r}_{i}-\boldsymbol{r}_{n-1, i}, \boldsymbol{e}_{n-1, i-1}={ }^{d} \boldsymbol{r}_{i-1}-\boldsymbol{r}_{n-1, i-1}, \boldsymbol{e}_{n, i-1}={ }^{d} \boldsymbol{r}_{i-1}-\boldsymbol{r}_{n, i-1}
$$


The time series of obtained error vector $\boldsymbol{e}_{n, i}$ are stored as error history ${ }^{(5)}$ then used for compensation of the output increment and joint input increment as follows ${ }^{(6)}$. The concept of this compensation is illustrated in Figure 1.

$$
\begin{aligned}
\Delta^{d} \boldsymbol{r}_{n, i} & =\Delta \boldsymbol{r}_{n-1, i}+\boldsymbol{e}_{n, i-1}-\boldsymbol{e}_{n-1, i-1}+\boldsymbol{e}_{n-1, i}, \\
\Delta \boldsymbol{\theta}_{n, i} & =J^{\#}\left(\boldsymbol{\theta}_{n, i-1}\right) \Delta^{d} \boldsymbol{r}_{n, i} \\
& =\Delta \boldsymbol{\theta}_{n-1, i}+J^{\#}\left(\boldsymbol{\theta}_{n, i-1}\right)\left(\boldsymbol{e}_{n, i-1}-\boldsymbol{e}_{n-1, i-1}+\boldsymbol{e}_{n-1, i}\right),
\end{aligned}
$$

where $C_{0}, C_{1}$ and $C_{2}$ are constant coefficients ranging $0<C_{0}<1,-1<C_{1}<0$ and $0<C_{2}<1$ to get converged solution, respectively. By substituting $\Delta^{d} \boldsymbol{r}_{n, i}$ of Eq.(6) into $\Delta \boldsymbol{r}_{m}$ of Eq.(4), the learning control scheme based on linear combination of error history is derived as

$$
\begin{aligned}
\Delta \boldsymbol{\theta}_{n, i}= & \Delta \boldsymbol{\theta}_{n-1, i}+J_{m}^{\#}\left(C_{0} \boldsymbol{e}_{n-1, i}+C_{1} \boldsymbol{e}_{n-1, i-1}+C_{2} \boldsymbol{e}_{n, i-1}\right) \\
& +\left(I-J_{m}^{\#} J_{m}\right)\left[J_{s}\left(I-J_{m}^{\#} J_{m}\right)\right]^{\#}\left(\Delta \boldsymbol{r}_{s}-J_{s} J_{m}^{\#} \Delta \boldsymbol{r}_{m}\right) .
\end{aligned}
$$

The proposed learning control scheme was applied to motion control of redundant planar serial manipulator in the case where the achievement of desired trajectory of the output link and the avoidance from obstacles are set as a main task and subtask, respectively ${ }^{(7)}$. Subtask vector $\Delta \boldsymbol{r}_{s}$ is set as the combination of each links' obstacle avoidance vector. An obstacle avoidance vector is given to make a link retreating the nearest obstacle with a velocity in inverse proportion to the distance between the link and the obstacle. Thus the obstacle avoidance vector $\boldsymbol{a}_{k, j}$ of the link $\boldsymbol{r}_{k}$ against to obstacle $\boldsymbol{O}_{j}$ is defined as

$$
\boldsymbol{a}_{k, j}=\frac{k_{\mathrm{a}}}{\left\|\boldsymbol{r}_{k}-\boldsymbol{O}_{j}\right\|+1} \frac{1}{1+e^{\left(d_{k, j}-D_{0}\right)}} \frac{\boldsymbol{r}_{k}-\boldsymbol{O}_{j}}{\left\|\boldsymbol{r}_{k}-\boldsymbol{O}_{j}\right\|},
$$

where $k_{\mathrm{a}}$ is repulsion coefficient, $d_{k, j}=\left\|\boldsymbol{r}_{k}-\boldsymbol{O}_{j}\right\|$ is the distance between the link and the obstacle, $D_{0}$ is the threshold of the area in which the avoidance velocity should be given. These variables are experimentally set to $k_{a}=1.0 \times 10^{-3}$ and $D_{0}=100 \mathrm{~mm}$, respectively. In Eq.(9), the amplitude of the obstacle avoidance vector is modified by using sigmoid function to make the avoidance velocity zero when the distance between objective link and obstacle is greater than $D_{0}$. From a number of avoidance vectors and Jacobian matrices of links having these vectors, the subtask vector $\Delta \boldsymbol{r}_{s}$ and its Jacobian matrix are defined as

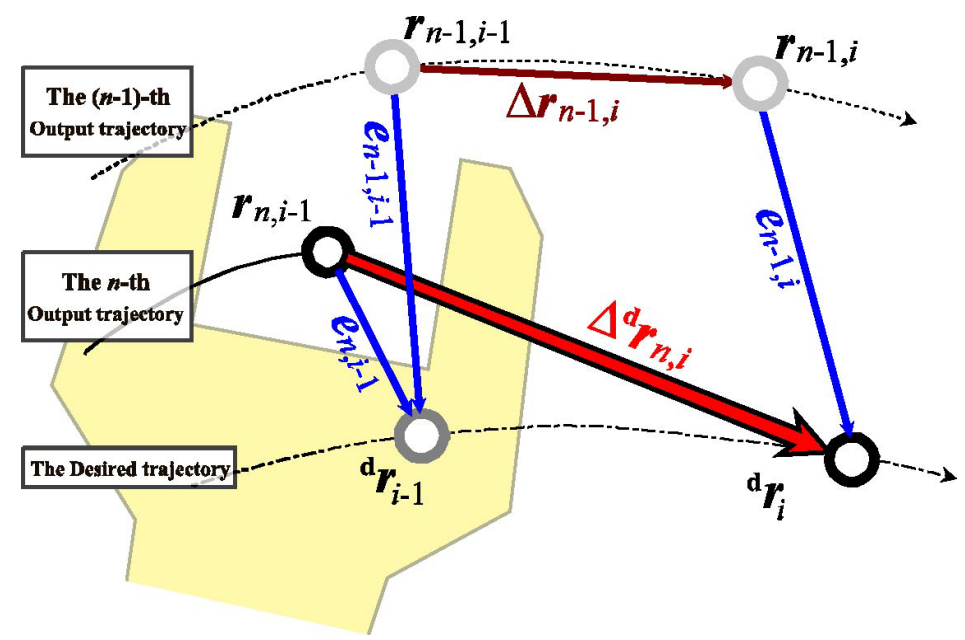

Fig. 1 Target trajectory and output error history 


$$
\Delta \boldsymbol{r}_{s}=\left(\begin{array}{lll}
\cdots & \boldsymbol{a}_{k, j}{ }^{\mathrm{T}} & \cdots
\end{array}\right)^{\mathrm{T}}, J_{s}=\left(\begin{array}{llll}
\cdots & J_{k}{ }^{\mathrm{T}} & \cdots
\end{array}\right)^{\mathrm{T}} .
$$

Motion control simulations were carried out with a planar serial 10R-manipulator which has ten links of $100 \mathrm{~mm}$, namely $1.0 \mathrm{~m}$ in full length, and based on the above-mentioned learning control scheme. The given initial configuration and obstacle settings are shown in Figure 2. When an obstacle is located at $(0.5,0.5)$, the 1 st position in Figure 2, robot can accomplish both of the tracing of linear desired trajectory from $(0.6,0.0)$ to $(0.6,0.5)$ and obstacle avoiding from the given initial configuration.

\subsection{Problems of the learning control on the application to hyper redundant robots}

Even though the learning control scheme formulated as Eq.(10) can work for simple case like the previous section, the two following problems should be discussed when we consider the actual application in more complex conditions.

(1) If robot is given many tasks without considering their harmonizing, desired trajectory cannot be achieved because of the confliction between each of them.

(2) The learning control scheme cannot modify initial configuration which is not suitable to achieve desired trajectory.

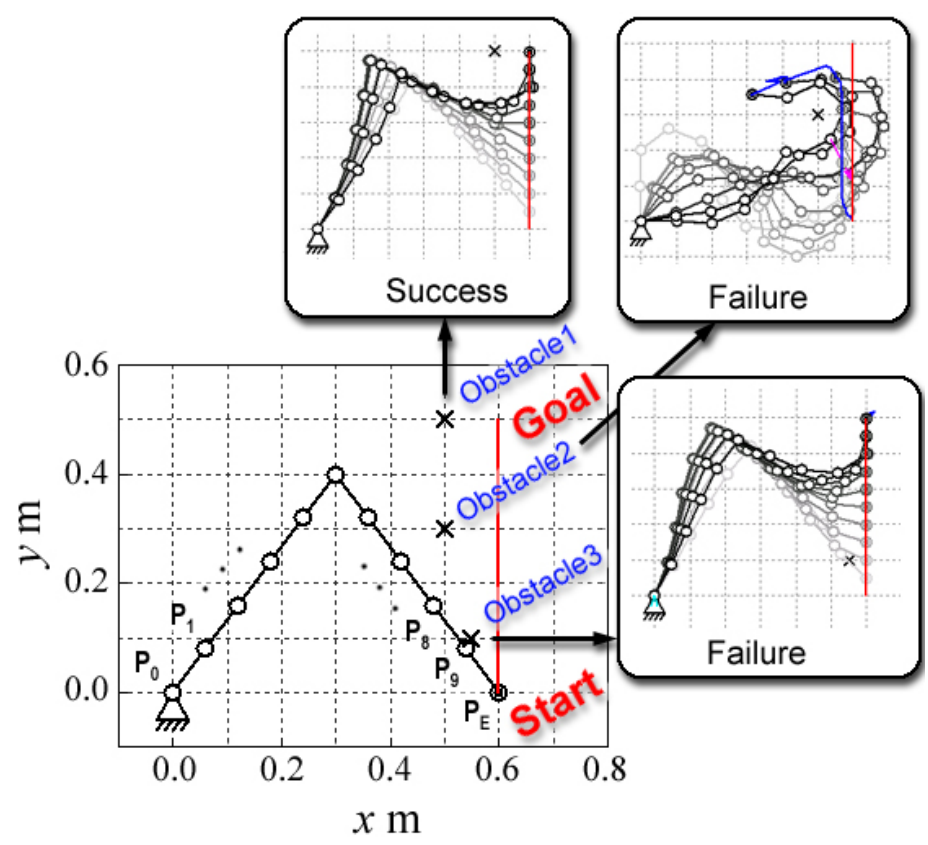

Fig.2 Given initial configuration, target trajectory, obstacle setting

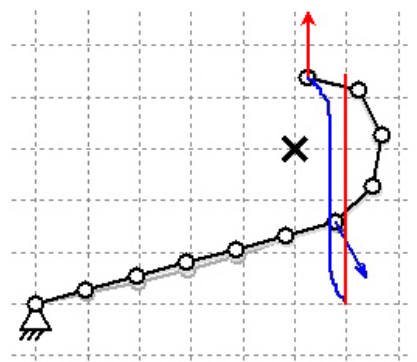

Configuration when $t=t_{i}$

(a) Partially singular configuration

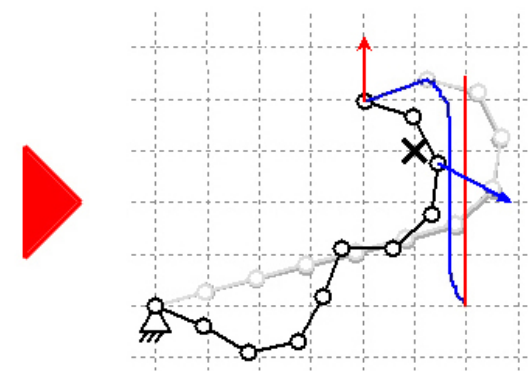

Configuration when $t=t_{i+1}$

(b) Failure in motion control

Fig.3 Undesirable rapid motion caused by partial singularity 
The first problem is seen when the obstacle is located on the 2nd position in Figure 2, $(0.5,0.3)$. In this case, hence the robot frequently generates rapid motion, the output link cannot achieve the desired trajectory. Figure 3 shows the configuration just before and after of rapid motion. As seen in this figure, part of manipulator, namely, several links from the fixed link aligned on a straight line. This means that the manipulator fell into a partial singular configuration before jumping. At that time, subtasks set on those links could not be accomplished while keep achieving the main task, therefore jumping motion was generated. In other words, because of interference of the main task and subtask, the manipulator took the partial singular configuration. If number of subtasks increased, the inference would occur easier and become a fatal problem for motion control of redundant manipulator. Improved subtask setting scheme thus should be necessary to reduce the interference.

The second problem is seen when the obstacle is located on the 3rd position in Figure 2, $(0.55,0.1)$. In this case, the robot cannot achieve the desired trajectory from the given initial configuration because it is too close to the obstacle. In addition, the summation of the distance between the anchor joint and the obstacle and the obstacle and the goal of the desired trajectory is almost same to the length of the manipulator. Namely, the robot cannot move without colliding the obstacle unless it starts moving from given initial configuration. The initial configuration thus should be modified by utilize the learning control scheme.

\section{Subtask setting scheme to improve achievement efficiency}

\subsection{Obstacle avoidance vector of vortex field}

In order to avoid the interference between the main task and the subtasks, a new concept to make a link passing through the side of obstacle, instead of retreating as possible, is proposed. As shown in Figure 4, two vectors are newly defined. One of them is $\boldsymbol{R}_{k, j}$ vector to retreat to a obstacle, corresponds to the previous obstacle avoidance vector $\boldsymbol{a}_{k, j}$, and the other is $\boldsymbol{W}_{k, j}$ vector which is perpendicular to $\boldsymbol{R}_{k, j}$ to pass through the obstacle. Then obstacle avoidance vector is redefined as $\boldsymbol{a}_{k, j}=\boldsymbol{R}_{k, j}+\boldsymbol{W}_{k, j}$. The vector $\boldsymbol{W}_{k, j}$ is formulated as

$$
\boldsymbol{W}_{k, j}=\left(\left\|\boldsymbol{r}_{k}-\boldsymbol{O}_{j}\right\| / D_{0}\right)^{2} \operatorname{sgn}\left(\Delta \boldsymbol{r}_{k} \cdot \hat{\boldsymbol{w}}\right) \hat{\boldsymbol{w}}\left\|\boldsymbol{R}_{k, j}\right\|,
$$

where $\hat{\boldsymbol{w}}$ is a unit vector given as

$$
\hat{\boldsymbol{w}}=\left[\begin{array}{cc}
0 & -1 \\
1 & 0
\end{array}\right] \frac{\boldsymbol{R}_{k, j}}{\left\|\boldsymbol{R}_{k, j}\right\|} .
$$

Eq.(11) defines the amplitude of $\boldsymbol{W}_{k, j}$ by its first term, then second and third term define the direction of the vector based on the dot product of $\Delta \boldsymbol{r}_{k}$ and $\hat{\boldsymbol{w}}$, respectively. This aligning of the obstacle avoidance vector direction to the main task vector direction reduces the interference between the main task and subtasks. This will improve the achievement efficiency of the entire tasks. The amplitudes of $\boldsymbol{R}_{k, j}$ and $\boldsymbol{W}_{k, j}$ are plotted against distance between objective link and obstacle in Figure 5. When a link is located near by an obstacle, $\boldsymbol{R}_{k, j}$ dominates the avoidance motion to make the link retreating the obstacle. In opposite, when a link is located far from an obstacle, $\boldsymbol{W}_{k, j}$ dominates the avoidance motion to make the link pass through the side of the obstacle. As the result, $\boldsymbol{a}_{k, j}$ forms a vector field with respect to the relative position of the link and obstacle as shown in Figure 6. Because the field looks like a vortex surrounding an obstacle, this scheme is called 'Obstacle avoidance vector of vortex field ${ }^{(8)}$. 


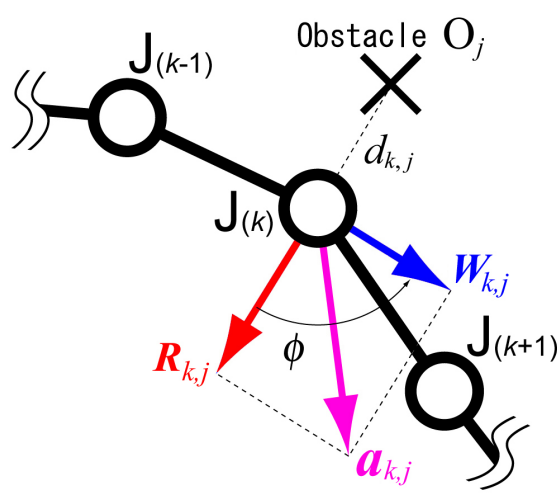

Fig.4 Definition of $\boldsymbol{R}_{k, j}$ and $\boldsymbol{W}_{k, j}$

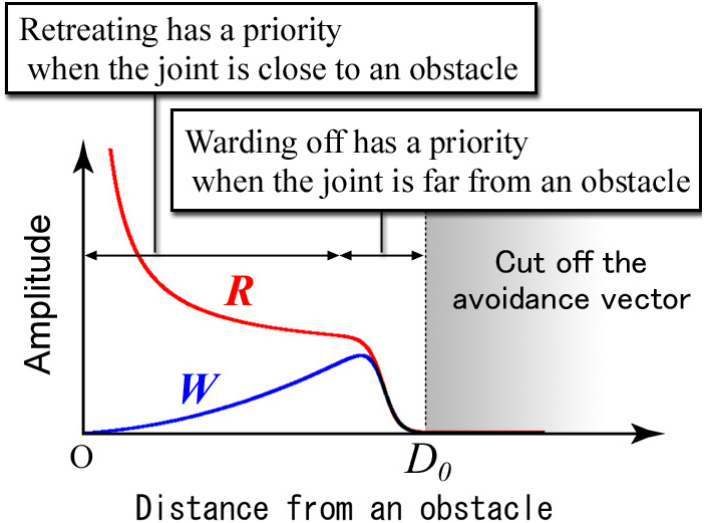

Fig.5 Amplitude of obstacle avoidance vectors

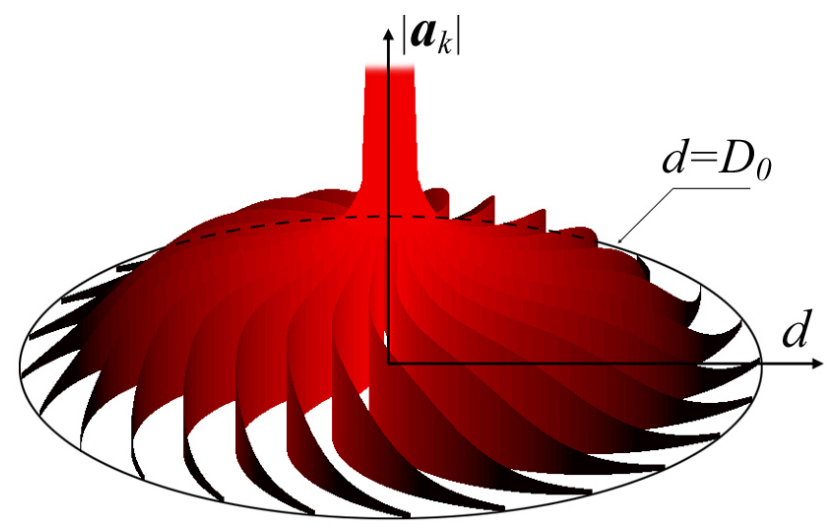

Fig.6 3D-visualized vortex field

\subsection{Subtask to assist the achievement of main task}

In the above-mentioned scheme, tasks have been given only for output link and links that close to obstacles. However, such elements cannot move freely because they are strongly restricted by desired trajectories or obstacles, and should be minor part when we mention hyper redundant robots. Therefore, lest of mechanism, which has never been considered, should be utilized with new subtask setting method to utilize hyper redundancy more effectively. In this section, new subtask setting concept to assist the achievement of main task is proposed. A main task assisting vector $\boldsymbol{h}_{k}$ is defined as

$$
\boldsymbol{h}_{k}=k_{h} \operatorname{sgn}\left[\Delta \boldsymbol{r}_{k} \cdot\left(\boldsymbol{r}_{m}-\boldsymbol{r}_{k}\right)\right] \frac{\boldsymbol{r}_{m}-\boldsymbol{r}_{k}}{\left\|\boldsymbol{r}_{m}-\boldsymbol{r}_{k}\right\|},
$$

where $k_{h}$ is a positive constant, $\Delta \boldsymbol{r}_{k}$ is a velocity vector generated by joint input to achieve main task. Eq.(13) denotes a velocity heading to the output link, when the direction from objective link to the output link is same to that of $\Delta \boldsymbol{r}_{k}$. In the contrary, if the direction from objective link to the output link is opposite to $\Delta \boldsymbol{r}_{k}$, Eq.(13) denotes a velocity to retreat from output link. By applying this new vector to assist main task on links far from obstacles, the objective links move in the same direction to that of the output link. This causes whole body motion of a hyper redundant mechanism so as to prevent from partial singularity then improves achievement efficiency. In the following simulations and experiments, the variable $k_{h}$ is experimentally set to $k_{h}=0.3 \times 10^{-3}$. 


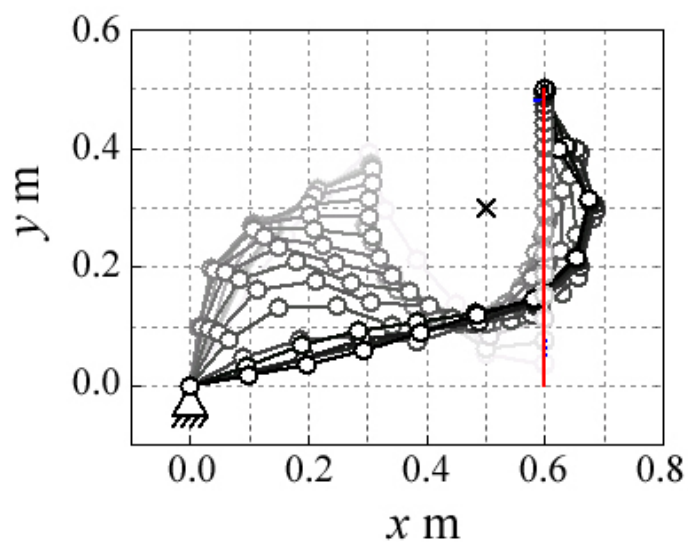

Fig.7 Improvement of the motion control

\subsection{Motion control based on the proposed subtask setting schemes}

The effectiveness of the proposed new subtask setting schemes is validated with a motion control simulation. Figure 7 shows the output trajectory when the obstacle is located on the second position in Figure 2 while given initial configuration and desired trajectory are same to Figure 2. The occasion of partial singularity is reduced and the 10R serial manipulator achieves the desired trajectory while avoiding the obstacle, though it could not be achieved by the previous learning control scheme.

\section{Acquisition of initial configuration with the backward learning}

To modify given initial configuration and acquire optimum one by the learning control scheme, a new method called 'backward learning' is proposed. The concept of the backward learning is shown in Figure 8. First, a manipulator acquires a desired trajectory with the conventional learning scheme which is hereafter called as 'forward learning'. Next, a new desired trajectory is given in opposite direction going from the final configuration to the initial configuration of the forward learning. Namely the final configuration of the forward learning is set as the initial configuration of the backward learning and the learning control based on the linear combination of error history is carried out again. Then the manipulator can acquire the final configuration of through a backward learning process, corresponds to the modified initial configuration of the forward learning. The forward and backward learning are cycled until the output error reduces less than the allowable amount, then a manipulator can acquire a desired trajectory including an optimized initial configuration.

To validate the backward learning scheme, motion control simulation in the 3rd case of Figure 2 is performed. From the given initial configuration in Fig.2, robot cannot achieve the desired trajectory without colliding in the first forward learning as shown in the Figure 9(a). By ignoring this collision, a converged solution can be obtained. Then a backward learning is carried out as shown in Figure 9(b) and a renewed initial configuration is obtained as shown in Figure 9(c). From this new initial configuration, the manipulator can achieve the desired trajectory while avoiding the obstacle as shown in Figure 9(d). This result supposes the effectiveness of the backward learning scheme. In the second simulation, the manipulator tries to achieve another desired trajectory in more complex environment. Figure 10 shows the given initial configuration, desired trajectory and obstacle arrangement. Through the same procedure to those above mentioned, the manipulator obtained improved initial configuration by performing a backward learning as shown in Figure 11. Figure 12 shows the output trajectory obtained from forward learning: (a) shows the trajectory when manipulator starts moving from the given initial configuration, (b) shows the trajectory from the improved initial configuration. The output 
error in the whole period of trajectory tracking of main task is evaluated by accumulated output error, calculated as $A E_{n}=\sum_{i=1}^{I} \boldsymbol{e}_{n, i}$. As shown in Figure 13, $A E$ was reduced from $10.96 \mathrm{~mm}$ to $8.95 \mathrm{~mm}$, namely by $21 \%$, by the backward learning.

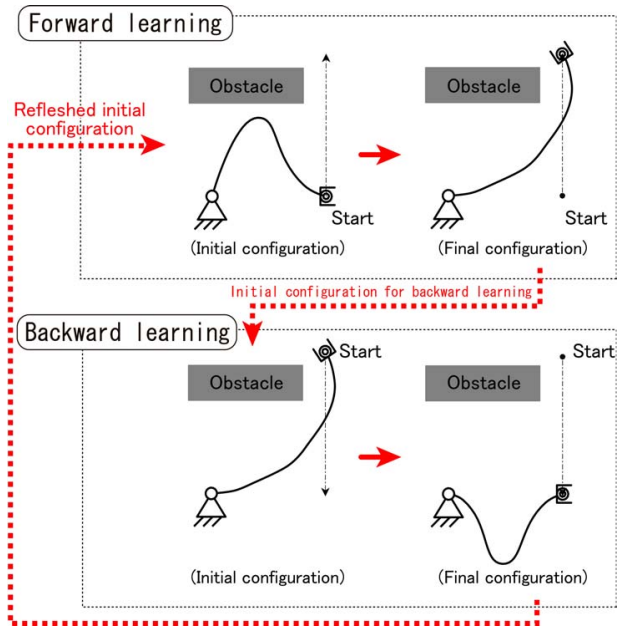

Fig. 8 Process of the backward learning scheme

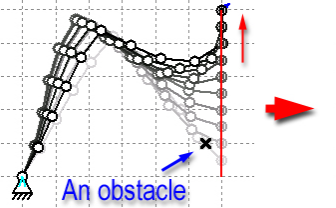

(a) 1st forward learning

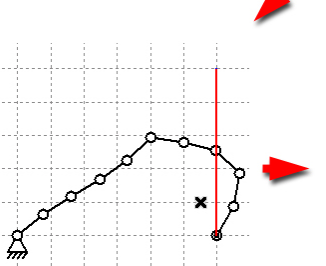

(c) Obtained starting configration

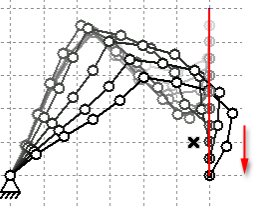

(b) Backward learning

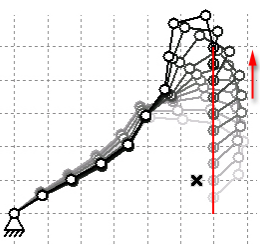

(d) Improved output motion

Fig.9 Achievement of desired trajectory with backward learning scheme

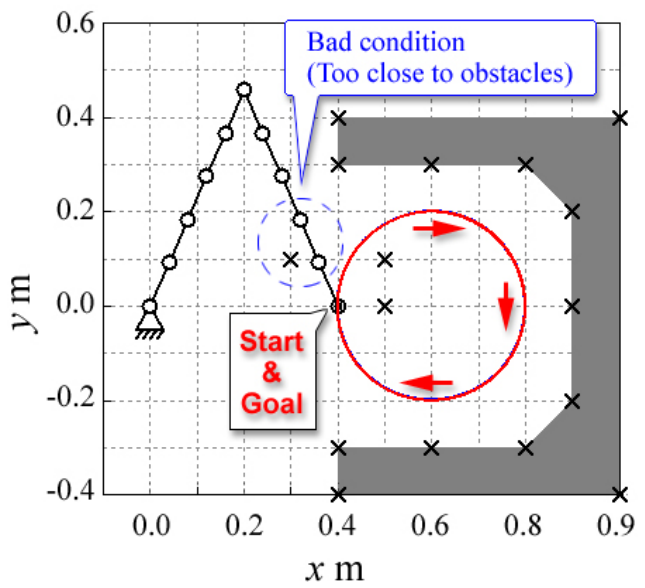

Fig.10 Given initial configuration and desired trajectory in a complex environment

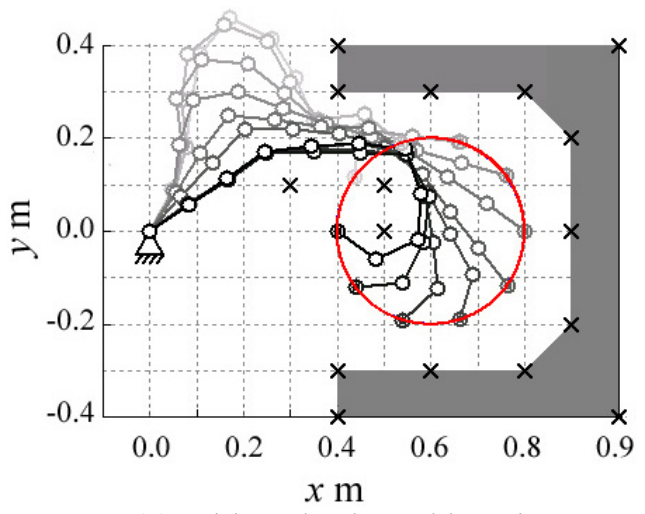

(a) Without backward learning

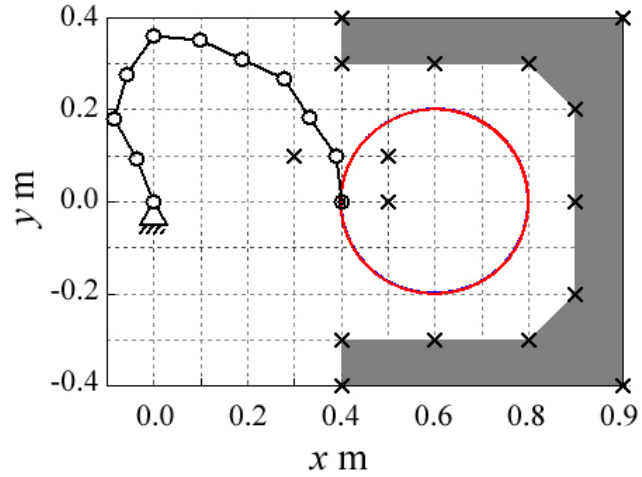

Fig.11 Obtained initial configuration by the backward learning scheme

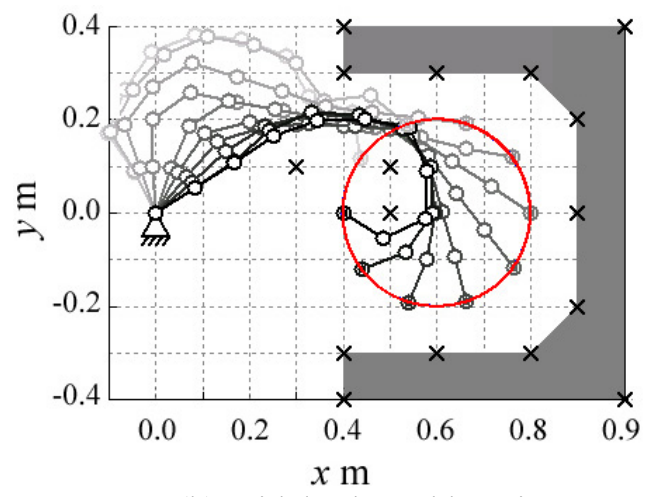

(b) With backward learning

Fig.12 Improvement of the motion control by the backward learning scheme 


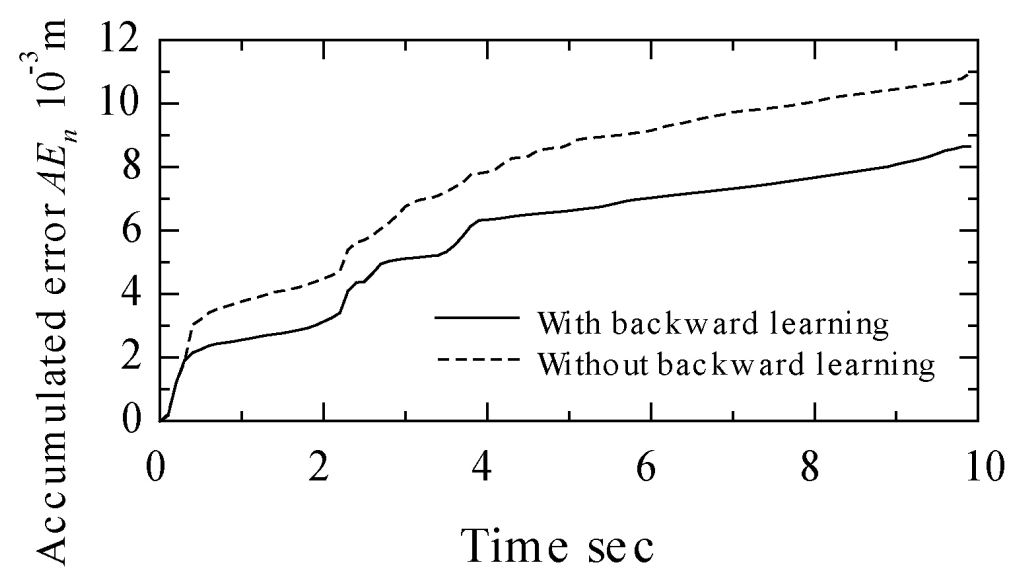

Fig.13 Decreasing of accumulated error by the backward learning scheme

\section{Simulations and experiments for validation}

Motion control experiments with a prototype are carried out. The prototype manipulator robot equipped 10 DC servomotors with a planetary reducer of which reduction ratio 159:1. A local servo controller based on RISC micro controller is attached to each motor and are connected to establish communications based on CAN2.0B serial protocol with a host computer which carries out the proposed learning control scheme. In the experiments, the arrangement of the desired trajectory and obstacles are the same as one in the simulation, shown in Figure 10. The converged solution of simulation is given as the initial trajectory to carry out experimental learning control. Figure 14(a) and (b) show the output trajectory obtained without and with learning control, respectively. Hence the output error is caused by unpredictable factors such as friction, saturation of motor output and gear backlashes, the prototype cannot achieve both of the desired trajectory tracking and obstacle avoiding without learning control. The experimental learning decreases those output error and make the prototype achieving the desired trajectory accurately while avoiding the obstacle. The accumulated output error $A E$ is reduced from $2.2 \mathrm{~m}$ to $0.7 \mathrm{~m}$, by $65 \%$ as shown in Figure 15 . This result demonstrates that the learning control reduces output errors caused by unexpected factors, and enables hyper redundant robots to accomplish the desired tasks in arbitrary complex environment.

\section{Conclusions}

In this paper, the learning control scheme based on error history is improved to control hyper redundant robots effectively. The obtained results are summarized as follows:

(1) After the formulation of the learning control, its two major problems on the application to hyper redundant robots, partial singular configuration problem and necessity of obtaining of optimum initial configuration, are revealed.

(2) By using the improved subtask setting schemes, obstacle avoidance vector of 'vortex field' and the main task assisting vector, redundant robots become able to achieve given tasks effectively without falling in the partial singular configuration.

(3) Based on the backward learning scheme, redundant robots can acquire the optimized initial configuration to achieve given tasks.

(4) Several motion control simulations and experiments demonstrate that planar redundant manipulator can generate the desired trajectory while avoiding obstacles. It is thus confirmed that the proposed method is effective and useful. 


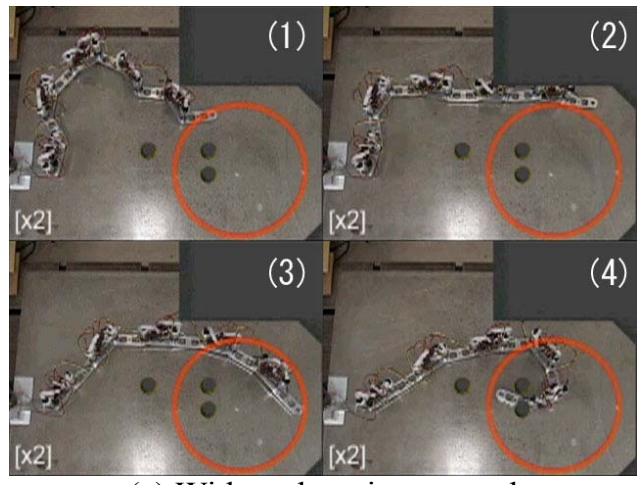

(a) Without learning control

)

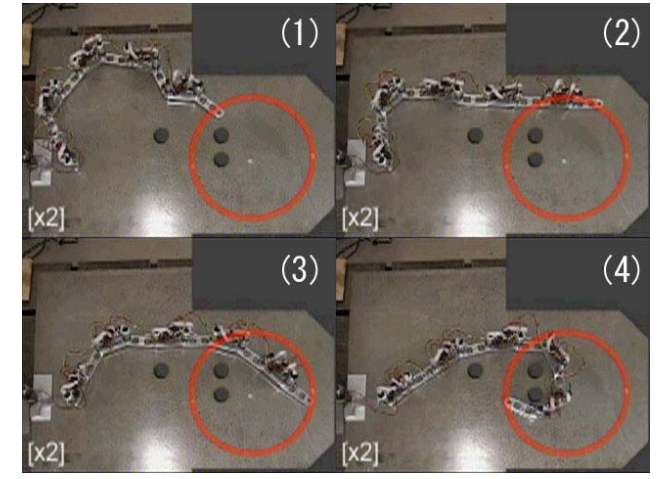

(b) With learning control

Fig.14 Obtained output trajectories in the experiments

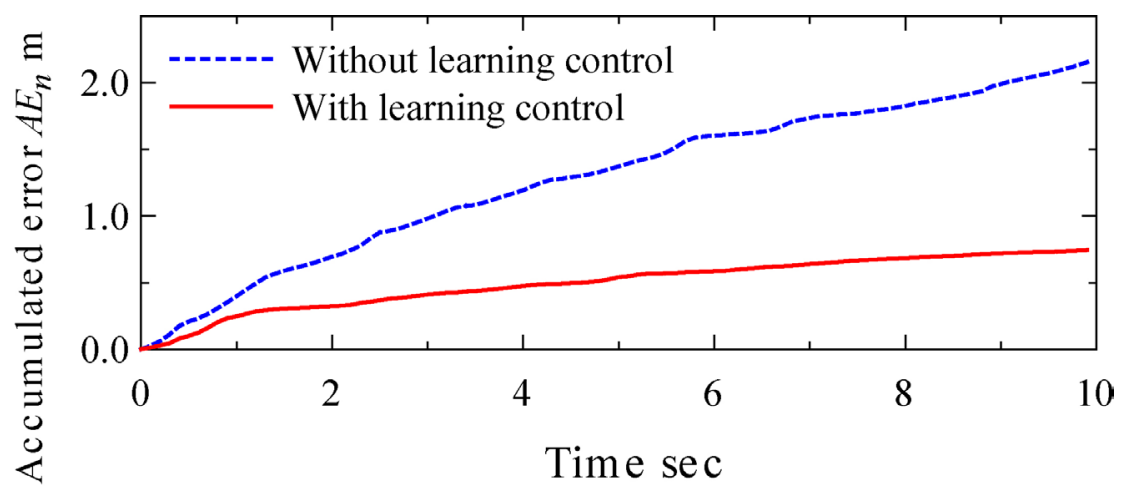

Fig.15 Output error obtained from the experiment

\section{References}

(1) M. Jamshidi and Z. Geng: Learning Control of Robot Manipulators, Expert Systems with Applications, Vol.4, (1992), pp. 297-304.

(2) S. Kawamura, F. Miyazaki and S. Arimoto: Realization of Robot Motion Based on Learning Method, IEEE Transactions on Systems, Man and Cybernetics, Vol.18, No.1(1988), pp. 126-134.

(3) W. S. Tang and J. Wang: A Recurrent Neural Network for Minimum Infinity-Norm Kinematic Control of Redundant Manipulators with an Improved Problem Formulation and Reduced Architecture Complexity, IEEE Transactions on Systems, Man, and Cybernetics. Part B: Cybernetics, Vol.31, No.1 , (2001), pp. 98-105.

(4) T. D. Sanger: Neural Network Learning Control of Robot Manipulators Using Gradually Increasing Task Difficulty, IEEE Transactions on robotics and automation, Vol.10, No.3, (1994), pp. 323-333.

(5) R. P. Roesser: A Discrete State-Space Model for Linear Image Processing. IEEE Trans. Automatic Control, AC-20, (1975), pp. 1-10

(6) J. Zhou, N. Iwatsuki, I. Hayashi and K. Morikawa: Learning Control of a Planar Mobile Robot of Serial Link Mechanism with Multi Degrees-of-Freedom, Proc. of JSME/JSPE Yamanashi Domestic Conf., (1999), pp. 43-44.

(7) D. Matsuura, N. Iwatsuki, Y. Shimada, K. Morikawa: Learning Control of Hyper Redundant Robots Based on Linear Learning Control Scheme, Proc. of the First Asia International Symposium on Mechatronics (2004), pp. 679-682.

(8) Daisuke Matsuura and Nobuyuki Iwatsuki: Motion Control of Hyper Redundant Robots by Use of Learning Control Based on Linear Combination of Error History, CD-ROM Proc. of The 9th International Conference on Mechatronics Technology, (2005), pp. 1-6. 